\title{
User Authentication by Secured Graphical Password Implementation
}

\author{
Ankesh Khandelwal \\ Department of Information \\ Technology and \\ Engineering \\ Heritage Institute of \\ Technology, \\ Anandapur, \\ Kolkata -700107, India
}

\author{
Shashank Singh \\ Department of Computer \\ Science and Engineering \\ Heritage Institute of \\ Technology, \\ Anandapur, \\ Kolkata -700107 , India
}

\author{
Niraj Satnalika \\ Department of \\ Instrumentation and \\ Engineering \\ Heritage Institute of \\ Technology, \\ Anandapur, \\ Kolkata -700107, India
}

\begin{abstract}
For the vast majority of computer systems, passwords are the method of choice for authenticating users. The most widely and commonly used authentication is traditional "Username" and "Password". For such authentication generally text (alphanumeric) is used. It is wellknown, however, that passwords are susceptible to attack: users tend to choose passwords that are easy to remember, and often this means that they are also easy for an attacker to obtain by searching for candidate passwords. On the other hand, if a password is hard, then it is often hard to remember. Keeping these things in mind we propose a novel, innovative and more secure way of selecting passwords: Graphical Passwords.

In this paper we explore an approach to user authentication that generalizes the notion of a textual password and that, in many cases, improves the security of user authentication over that provided by textual passwords. We design and analyze graphical passwords, which can be input by the user to any device with a graphical input interface.
\end{abstract}

We also try to answer two most important questions:

- "Are graphical passwords as secure and easy to use as text-based passwords"?

- "Major design and implementation issues for graphical passwords"?

The full text of the article is not available in the cache. Kindly refer the IJCA digital library at www.ijcaonline.org for the complete article. In case, you face problems while downloading the full-text, please send a mail to editor at editor@ijcaonline.org 